\title{
LA EDUCACIÓN PARA LA CIUDADANÍA: UNA URGENCIA EDUCATIVA PARA EL SIGLO XXI
}

\author{
Roberto Sanz Ponce ${ }^{1}$ \\ Ángela Serrano Sarmiento ${ }^{2}$ \\ Aurelio GonzÁlez Bertolín ${ }^{3}$
}

\begin{abstract}
RESUMEN: La escuela debe redefinirse para desarrollar capacidades personalesprofesionales-cívicas en los alumnos. El objeto de este artículo es describir el rol del docente, mediante un estudio exploratorio de tipo documental que analiza antecedentes y proyecciones futuras de una educación cívica. Esta se fundamenta en la adquisición de valores cívicos, mediante la vivencia personal y la imitación de la conducta docente. Por ello, el maestro debe poseer ciertas cualidades personales y pedagógicas; conocer estrategias metodológicas que desarrollen valores cívicos y dedicar tiempo para su asimilación y acomodación. Este enfoque replantea contenidos y políticas de selección y formación del profesorado, en base a estos interrogantes: ¿Qué tipo de sociedad queremos? y ¿Qué tipo de profesorado se necesita?
\end{abstract}

Palabras-clave: Profesorado. Educación para ciudadanía. Valores democráticos. Formación docente.

\section{EDUCATION FOR CITIZENSHIP: AN EDUCATIONAL URGENCY FOR THE $21^{\text {ST }}$ CENTURY}

ABSTRACT: The school must be redefined to develop personal-professional-civic abilities in the students. The purpose of this article is to describe the role of the teacher, through a documentary type exploratory study that analyzes the background and future projections of a civic education. This is based on the acquisition of civic values through personal experience and the imitation of the behavior of the teacher. Therefore, the teacher must possess certain personal and pedagogical qualities; know methodological strategies that develop civic values and dedicate time for their assimilation and accommodation. This approach rethinks content, and teacher selection and training policies, based on the following questions: "What kind of society do we want?" and "What type of teaching is needed?"

Keywords: Teachers. Education for citizenship. Democratic values. Teacher training.

1.Universidad Católica de Valencia - Departamento de Didáctica General, Teoría de la Educación e Innovación Tecnológica - Valencia, España. Email: roberto.sanz@ucv.es

2.Universidad Católica de Valencia - Departamento de Psicología, Educación inclusiva y Desarrollo Sociocomunitario - Valencia, España. Email: angela.serran@ucv.es

3.Universidad Católica de Valencia - Departamento de Didáctica General, Teoría de la Educación e Innovación Tecnológica - Valencia, España. Email: aurelio.gonzalez@ucv.es 


\section{EDUCAÇÂO PARA A CIDADANIA: UMA URGÊNCIA EDUCACIONAL PARA O SÉCULO XXI}

RESUMO: A escola deve ser redefinida para desenvolver habilidades pessoaisprofissionais-cívicas nos alunos. O objetivo deste artigo é descrever o papel do professor por meio de um estudo exploratório de tipo documental que analisa antecedentes e projeções futuras para uma educação cívica. Isso se baseia na aquisição de valores cívicos pela experiência pessoal e a imitação do comportamento docente. Portanto, o professor deve possuir certas qualidades pessoais e pedagógicas; conhecer estratégias metodológicas que desenvolvam valores cívicos e dedicar tempo para sua assimilação e acomodação. Essa abordagem repensa o conteúdo e as políticas de seleção e treinamento de professores, com base nas seguintes questões: "Que tipo de sociedade queremos?" e "Que tipo de professorado é necessário?"

Palavras-chave: Pessoal docente. Educação para a cidadania. Valores democráticos. Treinamento de professores.

\section{Introducción}

$\mathrm{E}$

1 desarrollo de la sociedad del conocimiento y el avance de las tecnologías de la información y de la comunicación deben hacernos replantear el sentido de la escuela (HARGREAVES, 2012). Sus fines, tradicionalmente, han estado relacionados con la adquisición de conocimientos. Estos saberes eran difíciles de adquirir por la población en general por ellos mismos. Pero, en la actualidad existen diferentes formas de acceso a un mundo inagotable e inabarcable de información.

También los sucesivos acontecimientos sufridos en Europa, fruto del fanatismo y la intolerancia, han llevado a los diferentes países miembros de la Unión Europea a establecer líneas de actuación preferentes en referencia a la educación. El 17 de marzo de 2015, los 28 ministros de educación firmaron la Declaración de París (COMISIÓN EUROPEA, 2016), en la que se comprometían a que los estudiantes adquiriesen las competencias cívicas, sociales e interculturales, promoviendo valores democráticos y derechos fundamentales, la inclusión social y la no discriminación, así como la ciudadanía activa. En 2017, fruto de esta creciente preocupación, esta misma Comisión comunicaba al Parlamento Europeo, al Consejo, al Comité Económico y Social Europeo y al Comité de las Regiones la importancia de situar a la educación como "la base de una ciudadanía activa [...] [que] contribuye a evitar el populismo, la xenofobia y la radicalización violenta" (COMISIÓN EUROPEA, 2017, p. 4), mediante la atención a la diversidad, el respeto a la dignidad humana y la concreción de valores comunes y compartidos.

Y para dar respuesta a estas demandas sociales, políticas y educativas, el papel del docente y de la escuela en la implementación de estos valores y capacidades, además del de la familia, es fundamental. ¿Qué papel puede y debe jugar la escuela ante esta nueva realidad?; ¿Qué contenido debe tener un enfoque de educación para la ciudadanía?; ¿Cuál es el rol que debe desempeñar el docente en la educación para la ciudadanía?; ¿Qué cualidades profesionales debe poseer? y ¿Cómo se debe seleccionar?

A lo largo de este artículo intentaremos responder a estas cuestiones, con el objetivo de plantear, teóricamente, las bases de una educación para la ciudadanía en la escuela, siendo conscientes de la existencia de otros factores y agentes que pueden potenciar o limitar esta educación. 


\section{¿Qué Papel Puede y Debe Jugar la Escuela ante esta Nueva Realidad?}

Diversos autores (NUSSBAUM, 2019; ROBINSON, 2015) afirman que los aprendizajes "formales" - curriculum académico - de la escuela obligatoria tienen poca transcendencia en su vida diaria y profesional. Estos autores demandan un giro en la concepción de la enseñanza, fundamentalmente en cuanto a qué deben aprender nuestros estudiantes (SANZ; SERRANO, 2017). Además, siguiendo la tradición de la Escuela Nueva y el aprendizaje proactivo, demandan metodologías activas y colaborativas, donde el foco del proceso de enseñanza-aprendizaje se sitúe en el alumno y se replanteen los modos y los escenarios educativos.

Es una constante en la literatura pedagógica actual situar al alumno en el centro del proceso educativo, preocuparse por sus intereses, atender sus dificultades de manera individual y personalizada, conocer sus conocimientos previos y cómo aprenden, para poder motivarlos y estimular su aprendizaje de manera significativa (MIGUEL; BLANCO, 2019), aunque, tampoco deberíamos obviar algunas corrientes pedagógicas que ponen en duda estos principios (ENKVIST, 2016).

En esa línea, España no parece desencaminada, ya que el informe TALIS (OECD, 2019) destaca un significativo incremento - entre 2008 y 2018 - en el porcentaje de profesores que afirman que el bienestar de los alumnos es importante; así como en el porcentaje de profesores que afirman que Si un alumno necesita ayuda el centro se la proporciona. Por tanto, se demuestra que el discurso y la propia práctica pedagógica caminan juntos (Tabla 1).

Tabla 1. Incremento en el interés por el bienestar del alumnado (2008-2018).

\begin{tabular}{cccccccc}
\hline \multirow{2}{*}{ Países } & \multicolumn{2}{c}{$\begin{array}{c}\text { La mayoría de los profesores de este centro cree } \\
\text { que el bienestar de los alumnos es importante }\end{array}$} & & \multicolumn{2}{c}{$\begin{array}{c}\text { Si un alumno de este centro necesita ayuda } \\
\text { extra, el centro se la proporciona }\end{array}$} \\
& $\mathbf{2 0 0 8}$ & $\mathbf{2 0 1 8}$ & Incremento \% & & $\mathbf{2 0 0 8}$ & $\mathbf{2 0 1 8}$ & Incremento \% \\
\hline Australia & 97,1 & 99 & 1,96 & & 91,6 & 94 & 2,6 \\
\hline Corea & 86,8 & 93 & 7,14 & & 85,6 & 83 & $-3,0$ \\
\hline Dinamarca & 98,1 & 99 & 0,92 & 82,1 & 76 & $-7,4$ \\
\hline Eslovaquia & 93,8 & 87 & $-7,25$ & 95,4 & 97 & 1,7 \\
\hline España & 93 & 96 & 3,23 & 86,9 & 91 & 4,7 \\
\hline Estonia & 93,8 & 98 & 4,48 & 95,7 & 98 & 2,4 \\
\hline Flandes (Bélgica) & 97,2 & 95 & $-2,26$ & 97,4 & 95 & $-2,5$ \\
\hline Italia & 94,8 & 96 & 1,27 & 85,8 & 90 & 4,9 \\
\hline México & 90,3 & 95 & 5,20 & 77,1 & 81 & 5,1 \\
\hline Noruega & 98,6 & 100 & 1,42 & 83,1 & 87 & 4,7 \\
\hline Portugal & 98,2 & 98 & $-0,20$ & 91,9 & 93 & 1,20 \\
\hline Promedio OCDE & 94,6 & 96 & 1,48 & 88,7 & 89,5 & 0,90 \\
\hline
\end{tabular}

Fuente: Adaptado de OCDE. Informe TALIS (OECD, 2019).

En ese sentido, todos los estudios coinciden en que los profesores más preocupados por el bienestar de los alumnos poseen un estilo docente más centrado en el alumnado. Así, por ejemplo, la OCDE (2015) evidenció como los centros escolares cuyos maestros expresaron preocuparse por el estado de bienestar de sus estudiantes, recibieron un mayor porcentaje de respuestas del alumnado que manifestaba sentirse feliz y haber encontrado un lugar en la escuela. 
Esa preocupación por el alumno, por su formación y desarrollo también se ve reflejada en un interés por educar ciertas capacidades personales, sociales y cívicas. La educación en valores, la educación del carácter y/o el desarrollo de capacidades así lo atestiguan. En cuanto a la educación para la ciudadanía, esta se ha convertido en un propósito moral; pero, para que se cumpla adecuadamente precisa de una forma distinta de Educación. Esta no se puede conseguir a través de asignaturas, ni tampoco mediante sermones ni charlas bienintencionadas, sino que requiere de un cambio en el clima y organización del centro y del aula, donde los alumnos expresen sus voces y sentimientos (SCHWARTZ; POLLISHUKE, 2015), se responsabilicen del aprendizaje; donde los docentes sean modelos de conducta y comportamiento ético, donde palabra y ejemplo se complementen, donde la educación adquiera su verdadero sentido (PUIG; MORALES, 2015). Por tanto, frente a una enseñanza teórica, "aprender el oficio de la ciudadanía" (LLANO, 2007, p. 100) y frente a una escuela dispensadora de conocimientos, una "auténtica escuela de ciudadanía” (GARCÍA RAGA; LÓPEZ MARTÍN, 2014).

Chomsky (2012), de manera crítica y, tal vez, injusta, sitúa el problema de la educación para la democracia y, por ende, para la ciudadanía ante una tesitura de difícil solución: "Lo cierto es que si una escuela tiene que imponer la enseñanza de los principios democráticos, hay razón para sospechar; cuanto más antidemocrática resulta ser una escuela, más necesidad siente de enseñar los ideales de la democracia" (CHOMSKY, 2012, p. 35). Aunque, seguramente injusta y desproporcionada, ¿tendrá algún matiz de realidad y/o coherencia esta afirmación?

\section{Hacia una Escuela más Humana. La Educación para la Ciudadanía}

¿La escuela prepara para la escuela, es decir, para superar los obstáculos que las asignaturas imponen; o prepara para el desarrollo personal, cívico, social - también académico? - ¿Qué capacidades desarrolla la escuela en los alumnos? Las Naciones Unidas, a través de su Informe para el Desarrollo (PNUD) afirmaba que la educación debe perseguir la libertad, la justicia y la paz en el mundo a través del desarrollo de la dignidad de todas las personas (FUKUDA-PARR, 2004). Años antes, el informe Aprender a Ser. La Educación del Futuro (FAURE, 1973) marcaba como objetivo fundamental "aprender a amar el mundo y a hacerlo más humano" (FAURE, 1973, p. 132), idea que fue retomada por el informe La Educación Encierra un Tesoro (DELORS, 1996), en el que se establecía el pilar: aprender a vivir juntos/a vivir con los demás. Estos informes proponían las líneas maestras de una educación para la ciudadanía. También, el último informe de la Unesco, Replantear la Educación. ¿Hacia un Bien Común Mundial? (BOKOVA, 2015), solicita una educación de carácter humanista, que respete la dignidad humana, la igualdad de derechos y la justicia social, mediante el respeto a la diversidad y la solidaridad, principios básicos para la ciudadanía. En ese sentido:

[...] parte del aprender a ser persona, en qué consiste la educación, es el aprendizaje para ser un agente moral y cívico en una comunidad: aprender qué es ser y vivir como miembro de esa sociedad; aprender a tomar parte en las variadas actividades teóricas y prácticas de la comunidad, a desempeñar el papel social que nos corresponde a cada cual como ciudadanos (NAVAL et al., 2017, p. 249).

Morín (2015), también afirma que la educación produce ciudadanos. Los ciudadanos producen democracia. Y la democracia favorece y permite una educación que forme ciudadanos. Pero, por desgracia, en todas las escuelas no prevalecen estos aprendizajes. ¿Quién decide lo que enseñar?; ¿Realmente se piensa en las capacidades que los alumnos van a necesitar a lo largo de su vida? y ¿Qué aspectos deberían aprenderse 
en la escuela para formar buenos ciudadanos? En líneas generales, se debe enseñar a convivir en sociedades diversas, donde se respete la diferencia. Dotar de capacidades personales para enfrentarse a un mundo complejo y cambiante y prepararles para ser ciudadanos informados, activos y comprometidos (PÉREZ, 2016).

El "humus" de esa educación para la ciudadanía debe ser la enseñanza de las Humanidades (NUSSBAUM, 2019), si no corremos el peligro de que en lugar de ciudadanos cabales, reflexivos, críticos y preocupados por el otro, produzcamos generaciones de "máquinas utilitarias" o de formar una "visión clientelar de la ciudadanía" (GUICHOT, 2012, p. 36). Pero las Humanidades van desapareciendo del currículum académico progresivamente, frente a un conocimiento más técnico y utilitarista, que responde a la lógica del mercado. En esa escuela más "humana," los valores cívicos a enseñar son la libertad, la igualdad, la solidaridad, el respeto activo y el diálogo, a los que se podrían añadir la participación, la justicia, la tolerancia y la responsabilidad; junto con las capacidades de escuchar, dialogar y participar y las aptitudes para reflexionar, reconocer al otro, interesarse por su vida, imaginar, emitir juicios críticos, pensar en el bien común (GIROUX, 2019). Todo ello, en un ambiente de libertad. Pero, nos encontramos con ciertas dificultades a la hora de educar la libertad, ya que no debemos olvidar que tanto la libertad como la igualdad siempre se encuentran amenazadas, por lo que el papel del docente es fundamental en la implementación y gestión de ambos valores.

Además, en esta nueva escuela debemos esperar que los alumnos sepan "reconocer al otro, clave para que el otro también los reconozca, sepa responder en la sociedad en la que vive de forma autónoma y personal. Sepa ser él mismo y sea capaz de ser un ciudadano que sabe asumir sus deberes para con la sociedad y capaz de participar en ella" (GARCÍA ARETIO et al., 2011, p. 211). Por tanto, debemos perseguir una escuela menos preocupada por el "currículum oficial," por enseñar solo para la sociedad del conocimiento y más preocupada en "enseñar más allá de ésta, de modo que se aborden otros valores humanos [...] relacionados con el carácter, la comunidad, la democracia y la identidad cosmopolita" (HARGREAVES, 2012, p. 73), en la misma línea que propone Hannah Arendt (1961), cuando define la educación como aprender a comprender el mundo y a desarrollar la capacidad de elaborar juicios.

\section{El cómo y el dónde de la educación para la ciudadanía}

La educación para la ciudadanía solo se consigue cuando el alumno "se inserta en un ethos, es decir, en un ambiente fértil, moralmente denso, humanamente acogedor” (LLANO, 2007, p. 100). La educación para la ciudadanía solo puede ser aprendida desde un aprendizaje vivencial, no por ley ni por castigo. Debe ser aprendida desde la experiencia personal; desde el aprendizaje en los primeros años (NOVELLA et al., 2013); desde un estilo docente democrático; desde una distribución del mobiliario en el aula que permita la participación, las relaciones personales, el trabajo en equipo; y donde los tiempos destinados a la reflexión colectiva (DAY, 2018) beneficien la adquisición de las virtudes cívicas. Y todo ello desde la riqueza de la diferencia, donde la escuela inclusiva es garante de la educación para la ciudadanía global.

Tal como establece la Unesco (2017), la escuela actual presenta grandes flujos de población provenientes de todo el mundo que son un rasgo característico del mundo moderno. Esta diversidad significa que las escuelas atienden a niños de diferentes culturas -heterogeneidad cultural-, considerándose como una oportunidad de crecimiento personal, de respeto a la diferencia y de educación para la ciudadanía. Bajo esta situación, se requiere que todos los niños se mezclen y aprendan de otras culturas. Lejos de difuminar la diversidad cultural de los alumnos, la educación para la ciudadanía puede resaltar el valor de las diferencias, respetando y afirmando la universalidad de los principios de los Derechos Humanos. El respeto por los demás significa, en la vida escolar, un diálogo con los demás y un interés en otros estilos de vida familiares, hábitos sociales y prácticas culturales. La educación para la ciudadanía es el foro ideal que 
ayuda a expresar opiniones sobre diferentes formas de mirar el mundo, en otras palabras, sobre las diversas culturas y la necesidad de desarrollar capacidades para interactuar y crecer juntos en un mundo cada vez más globalizado. Con ello, no estamos afirmando que sea fácil abordar esta educación en la diversidad, ni que la gestión del aula y del aprendizaje no se complejicen, simplemente mantenemos que se trata de uno de los fines de la educación y que se corresponde con un derecho fundamental de todos los estudiantes.

Esta educación debe perseguir el desarrollo de la dignidad humana; fomentar el conocimiento, reconocimiento y vivencia de los Derechos Humanos; promover la autonomía personal y el desarrollo de capacidades; favorecer la igualdad y la inclusión; implementar una democracia vigorosa; humanizar a los educandos y perseguir la justicia social (SANZ; SERRANO, 2016).

Pero, tal y como se aprecia en el informe TALIS (OECD, 2019), respecto a las metodologías utilizadas por el profesorado en España, los docentes han mejorado de manera significativa en el uso de metodologías reflexivas tales como: estimular el pensamiento crítico o el uso de paralelismos con la vida cotidiana; pero muestran estar por debajo de la media de la OCDE en el uso de metodologías activas que estimulen la autonomía del alumnado, como pueden ser: el uso de las nuevas tecnologías o el trabajo en pequeños grupos (Fig.1). De manera similar al anterior informe (MECD, 2014) se observa una necesidad de

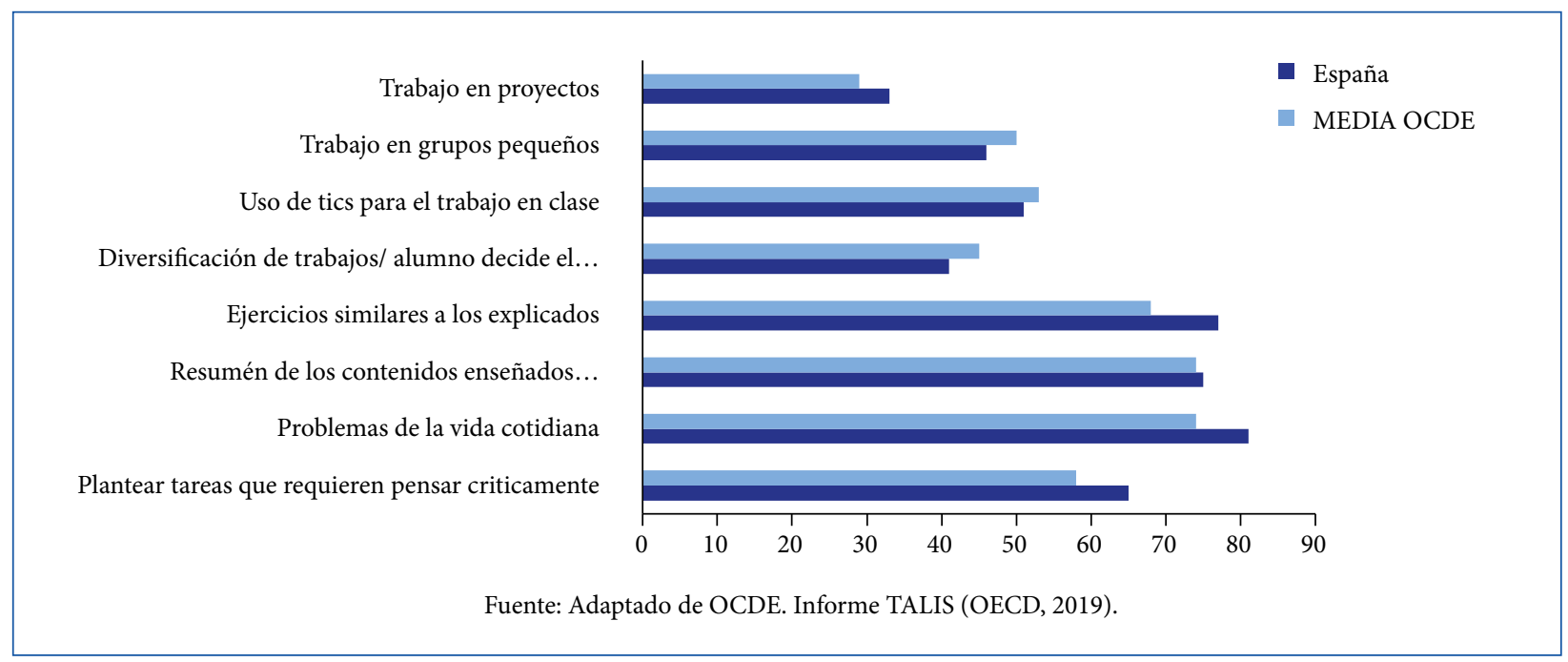

Figura 1. Metodologías utilizadas por el profesorado en España y Media OCDE.

estimular metodologías que consideran al alumno como sujeto de su propia formación.

Sin embargo, tal como establece el informe Measuring Global Citizenship Education (UNESCO, 2017), si realmente se quiere formar en una ciudadanía para el siglo XXI, que atienda a la inclusión de la diversidad en un mundo globalizado, el currículum actual debería tender a la formación en las siguientes competencias:

1. Empatía.

2. Pensamiento crítico y resolución de problemas.

3. Capacidad de comunicarse y colaborar con otros.

4. Resolución de conflictos.

5. Sentido e identidad personal.

6. Valores universales compartidos (Derechos Humanos, paz, justicia, etc.).

7. Respeto por la diversidad/comprensión intercultural.

8. Reconocimiento de problemas globales: interconexiones (ambiental, social, económica, etc.). 
El reto de responder a estas competencias mediante la educación supone una tarea compleja en la que han de redefinirse los principios a seguir en una educación global y no segmentada. Esto supone, por tanto, el análisis pormenorizado de las tendencias, los recursos y los fines actuales del sistema educativo y no perder de vista la influencia de la familia y la necesidad de unificar criterios.

\section{¿Qué Contenido Debe Tener un Enfoque de Educación para la Ciudadanía?}

El International Civic and Citizenship Education Study (SCHULZ et al., 2018), realizado en 38 países con 140.000 estudiantes y 62.000 maestros, propone los dominios y contenidos claves de una educación para la ciudadanía. A modo de recopilatorio, estos contenidos podrían presentarse de la siguiente manera:

Tabla 2. Contenidos globales de educación para la ciudadanía.

\begin{tabular}{|cccc|}
\hline & \multicolumn{2}{c|}{ Contenidos } \\
\hline $\begin{array}{c}\text { Conglomerado 1 } \\
\text { Sociedad cívica y sistemas }\end{array}$ & $\begin{array}{c}\text { Conglomerado } 2 \\
\text { Principios cívicos }\end{array}$ & $\begin{array}{c}\text { Conglomerado 3 } \\
\text { Participación cívica }\end{array}$ & $\begin{array}{c}\text { Conglomerado 4 } \\
\text { Individuo e Identidades } \\
\text { cívicas }\end{array}$ \\
\hline Los ciudadanos & Equidad & Toma de decisiones & $\begin{array}{c}\text { El sujeto frente a la vida } \\
\text { cívica }\end{array}$ \\
\hline $\begin{array}{c}\text { Las instituciones del } \\
\text { Estado }\end{array}$ & $\begin{array}{c}\text { Libertad y Derechos } \\
\text { Humanos }\end{array}$ & $\begin{array}{c}\text { Influencia y consecuencias } \\
\text { de la participación }\end{array}$ & $\begin{array}{c}\text { Interacciones cívicas } \\
\text { como sujeto } \\
\text { (vida en comunidad) }\end{array}$ \\
\hline $\begin{array}{c}\text { Las instituciones civiles } \\
\text { Reglas y leyes }\end{array}$ & Sentido comunitario & $\begin{array}{c}\text { Participación en la vida } \\
\text { comunitaria }\end{array}$ & \\
\hline
\end{tabular}

Fuente: Adaptado de SCHULZ et al. (2016; 2018).

Cada uno de estos contenidos, tal como establece el estudio de Martin y Mullis (2013), debe ser fundamentado desde dos dominios y dos perspectivas de cada dominio. Un dominio cognitivo a nivel de conocimientos:

Dominio cognitivo 1: El manejo y conocimiento conceptual.

Dominio cognitivo 2: Razonamiento y aplicación práctica de tal contenido.

Y un dominio afectivo-comportamental, propuesto por Schulz, Ainley, Fraillon, Kerr y Losito (2011), para una aplicación práctica.

Dominio afectivo-comportamental 1: Crear una actitud responsable.

Dominio afectivo-comportamental 2: Incentivar un compromiso social.

Esta propuesta de contenidos no sólo plantea la necesidad de formar a los alumnos desde aspectos conceptuales, epistemológicos y sociales, sino que representa la necesidad de preparar al profesorado para este tipo de formación. Según los datos de la Fundación CIVES (2015), el 89,5\% del profesorado reconoce esta necesidad. Además, tal como expone la Unesco (2016), dentro del marco de los retos de la educación para el 2030, no sólo se ha de focalizar la educación en la ciudadanía pensando en una parte de sistema educativo currículo explícito - , sino que se ha de partir de la idea de que el docente es responsable de la formación de 
futuros ciudadanos y su trabajo reclama la misión de educar en la responsabilidad social y el impacto de las consecuencias de sus acciones en la vida democrática de un país - currículo implícito.

La educación para la ciudadanía, por tanto, está necesariamente vinculada a una posición ética y comprometida del profesorado. Es, por esa razón, de vital importancia clarificar: ¿quién es el profesorado idóneo para este tipo de enfoques y qué exigencias competenciales debe tener? Desde esta perspectiva la palabra tutor de formación en la ciudadanía cobra una clave epistemológica específica. Es decir, que el docente asume la responsabilidad social de formar nuevos ciudadanos desde una ética profesional y personal. Este tipo de enfoque exige ver al profesorado como un profesional decisivo en la consecución de los objetivos educativos y, por tanto, tanto la formación como la carrera docente, exigen cambios estructurales.

\section{¿Cuál es el Rol que Debe Desempeñar el Docente en la Educación para la Ciudadanía?}

Informes internacionales manifiestan que para conseguir buenos resultados en la educación se precisa contar con buenos docentes, directores, orientadores e inspectores. Concretamente, el informe Mckinsey (BARBER; MOURSHEED, 2007) señala que el nivel educativo de un país depende de la formación, motivación, valores y aprendizaje permanente de sus profesores.

Tal como establece la Unesco (2016), existe una tendencia en educación a creer que la única necesidad de los recursos personales de los centros, está dirigida a cubrir la oferta y la demanda de población escolar en referencia a la ratio alumno-profesor. Sin embargo, en países con resultados positivos en PISA se pone de manifiesto que esto no es así. En Suecia o Finlandia, por ejemplo, con buenos resultados no sólo académicos, sino también competenciales y actitudinales, la proyección de una escuela para el siglo XXI evidencia que no sólo es importante la contratación de profesorado que cubra la ratio por alumnos, sino fundamentalmente de profesorado especializado y formado en todas aquellas competencias y valores que garantizan la calidad educativa, para poder responder a los actuales retos de la educación.

El informe TALIS (MEFP, 2019) evidencia que en España se redujo significativamente el tiempo dedicado al mantenimiento del orden en clase, de 2008 (15,7\%) a 2013 (14,7\%), pero que ha aumentado en 2018 (16,4\%). Este aumento nos aleja de la media de los países de la OCDE (13,4\%). Tal como se observa en la siguiente figura, elaborada a partir del informe TALIS (OECD, 2019), queda evidenciada la necesidad de formación del profesorado en aspectos tales como: formación en las competencias que imparte; formación pedagógica específica de su especialidad; formación en atención a la diversidad; y en otras competencias, ya no sólo disciplinares, sino en ámbitos como la resolución de conflictos, la gestión del aula o la educación en valores. Los resultados de España comparados con los de la media de los países de la OECD son significativamente menores, lo que evidencia la necesidad de formación (Fig. 2).

Estos datos reflejan la necesidad de actualización en el currículo de formación de los docentes para responder a los retos del siglo XXI, así como medidas para atraer a la profesión docente a los mejores candidatos. Es decir, convertir los estudios de Magisterio y de Formación del Profesorado en titulaciones atractivas para los estudiantes más brillantes de la Enseñanza Media. Para ello, es necesario fortalecer su imagen social y ofrecer condiciones profesionales, económicas y de promoción suficientemente incentivadoras. 


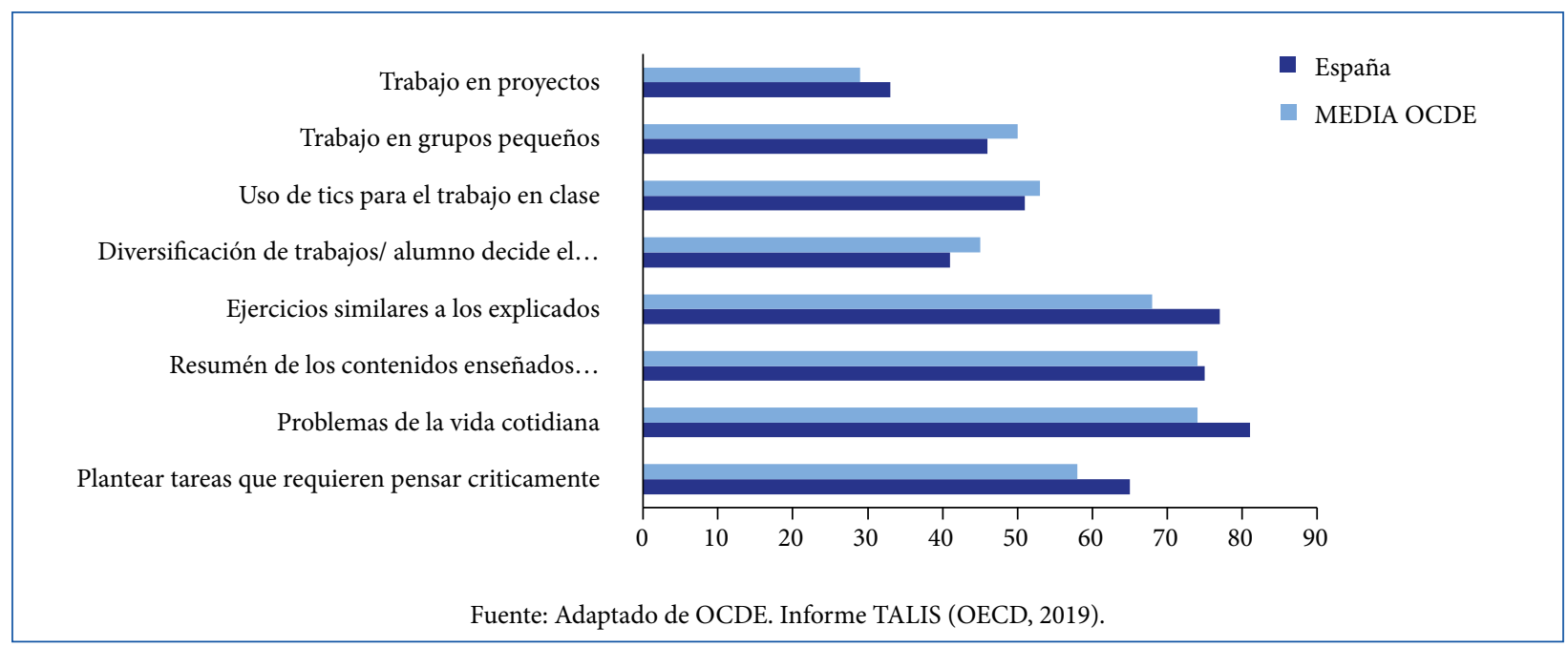

Figura 2. Porcentaje de profesores de Educación Secundaria Obligatoria que indican tener necesidad de formación en otros aspectos educativos. España y Media OCDE.

\section{Qué Cualidades Profesionales debe Poseer el Docente}

La Carta del Consejo de Europa sobre la educación para la ciudadanía democrática y la educación en Derechos Humanos (CONSEJO DE EUROPA, 2010) dedicaba un apartado a la formación del profesorado y establecía la necesidad de implementar principios democráticos y prácticas cívicas, tanto en el proceso formativo como en el posterior desarrollo profesional de los docentes, para asegurar la continuidad y la eficacia de la educación para la ciudadanía. La Fundación CIVES (2015), en ese sentido, recogía el sentimiento generalizado $(98,2 \%)$ del profesorado acerca de las medidas y recomendaciones elaboradas por el Consejo de Europa, así como la necesidad de crear materiales curriculares específicas $(87,7)$ para la implementación de una adecuada educación para la ciudadanía.

El perfil profesional deseable del profesorado del siglo XXI -aun siendo consciente de otras clasificaciones (PRING, 2016; LÓPEZ-LUJÁN, 2018)- podría definirse en razón de una serie de rasgos, competencias y cualidades profesionales. La mayoría de las clasificaciones acerca de las competencias docentes recogen la importancia del desarrollo de cualidades y/o capacidades de índole social y cívico entre el profesorado. Del mismo modo, el aspecto ético también se encuentra presente en casi todas las clasificaciones. Para este artículo y como propuesta para la reflexión, agrupamos las competencias en torno a los cuatro pilares de la educación para el siglo XXI:

1. Saber: estar en posesión de una amplia cultura general y dominar los conocimientos propios del área que imparte. Así como disponer de una sólida formación didáctica y pedagógica.

2. Saber hacer: saber planificar, desarrollar y evaluar el proceso de aprendizaje-enseñanza de los estudiantes. Usar y aplicar con pertinencia y responsabilidad un amplio repertorio de recursos didácticos, especialmente los vinculados a las tecnologías de la información.

3. Saber convivir: estimular el esfuerzo del alumnado y promover su capacidad para aprender por sí mismos y en colaboración con otros compañeros. Tener capacidad para el diálogo con todos los miembros de la Comunidad Educativa. Adoptar una actitud de empatía 
con el alumno y sus familias; mantener actitudes que fomenten un clima de colaboración, convivencia, respeto y libertad responsable.

4. Saber ser: tener autoestima, conocimiento de las propias capacidades, equilibrio emocional y autocontrol. Estar en posesión de una clara identidad docente y sentirse profesor por encima de cualquier otra consideración, entendiendo que el profesor ha de ser, al mismo tiempo, educador y tutor de sus alumnos. En definitiva, tener vocación docente y disfrutar de la profesión.

Para lograr la consecución de dicho perfil debe asegurarse entre el profesorado una serie de condiciones que dignifiquen la profesión. Sin embargo, esta propuesta resulta chocante al analizar los datos del informe TALIS (MEFP, 2019), en el que se refleja que una buena parte de los profesores no se identifican con este perfil, fundamentalmente por las condiciones en las que desarrollan su trabajo. Los docentes de Educación Secundaria en España identifican una serie de impedimentos a la hora de participar en actividades de desarrollo profesional. Un $10 \%$ afirma que no cumple con los requisitos previos; un 42\% mantiene que son demasiado caras; un $29 \%$ no encuentra el apoyo de sus superiores para realizarlas; un 59\% las ve incompatibles con su horario laboral; un $58 \%$ piensa que no tiene tiempo; un $54 \%$ afirma que la oferta formativa es inadecuada; y un $76 \%$ cree que no existen incentivos para el desarrollo profesional de los docentes.

Teniendo en cuenta estas cuestiones evidenciadas en las respuestas del profesorado evaluado, valdría la pena preguntarse con qué recursos se cuenta para atraer a los mejores profesionales para ejercer la docencia, siendo conscientes de la gran responsabilidad social que se le demanda a la educación y su profesorado.

\section{Cómo se Debe Seleccionar al Profesorado}

La Formación del Profesorado en España constituye un modelo consecutivo, de cuatro + uno, que ofrece una formación didáctica y pedagógica manifiestamente insuficiente (GONZÁLEZ BERTOLÍN; SANZ, 2014; MARINA, 2017). En los tramos obligatorios del sistema educativo -y en Educación Infantil-, donde la formación didáctica y pedagógica es tan relevante como el dominio de la episteme, sería deseable un modelo de formación simultáneo, es decir, un Grado. Un Grado de Maestro de Infantil, un Grado de Maestro de Primaria y un Grado de Profesor de Educación Secundaria Obligatoria.

Los sistemas de selección del profesorado no universitario reclaman una reforma sustancial del modelo de selección del profesorado. El modelo actual no sirve para seleccionar a los mejores docentes, sino, en el mejor de los casos, a los mejores opositores. Y no siempre (GONZÁLEZ BERTOLÍN; SANZ, 2016). En cualquier caso, es un proceso selectivo en el que se pretende valorar el saber, en menor medida el saber hacer, pero que no aporta evidencia alguna sobre el saber convivir y el saber ser. La selección del profesorado debería estar más estrechamente relacionada con el logro de los estudiantes en la formación inicial y, sobre todo, con la evaluación de evidencias de sus buenas prácticas y compromiso ético con la profesión (MEIRIEU; MONTENEGRO, 2019), todo ello si pretendemos que la escuela dé respuesta a las demandas sociales de una sociedad más democrática y de ciudadanos con mejores y más asentados valores morales, políticos y cívicos. El papel de los mentores pedagógicos (MARINA et al., 2015), docentes con más experiencia que acompañan a los profesores noveles en los aspectos personales, sociales y profesionales podría ser una buena solución. Ese acompañamiento personal y asesoramiento pedagógico, debiera concluir con una evaluación de esa iniciación en la carrera docente, señalando los puntos fuertes y débiles del profesor novel y orientado su formación continua en consecuencia. En cualquier caso, informes Internacionales (BARBER; MOURSHEED, 2007) afirman que en los países con mejores resultados educativos la selección del profesorado es muy exigente. 


\section{Consideraciones Finales}

La ciudadanía es un constructo elaborado por el ser humano como ser social y como tal debe ser educado. A ser ciudadano se aprende (PÉREZ, 2016), por ello es necesario una educación para la ciudadanía. "Los niños/as no son ciudadanos/as del futuro, sino ciudadanos/as del presente [...], la mejor manera de formales es empezar por considerarles y tratarles como ciudadanos de verdad y no como embriones de ciudadanos" (NOVELLA et al., 2013, p. 93-94).

Tratar a los niños como ciudadanos de verdad significa desarrollar el valor de la responsabilidad social en todos sus actos, identificando que cada derecho se cimienta sobre un deber y que ser sujetos de derechos nos obliga a respetar los derechos sociales y los derechos de los otros.

\section{¿Qué tipo de ciudadanos se quieren conseguir con una educación para la Ciudadanía?}

Se trata de una educación que promueva el reconocimiento del otro, que favorezca la construcción de un proyecto común, que no excluya y atienda a todos (MÍNGUEZ; ROMERO, 2018; BUXARRAIS, 2018), más, si cabe, en un momento como el actual en el que proliferan las iniciativas disgregadoras, populistas y localistas. También es necesario, si pretendemos construir una sociedad verdaderamente democrática y evitar los riesgos y peligros de una población desinformada e ineducada, desarrollar entre los jóvenes las capacidades cívicas, políticas y sociales. La educación:

[...] ha de enfatizar, junto al cuidado de la 'alfabetización política' y de la participación en la vida pública, social y cultural, el desarrollo del pensamiento crítico y de las actitudes que promueven la concienciación y la responsabilidad de reconocerse como ciudadanos (BERNAL et al., 2017, p. 102).

\section{¿Qué tipo de profesorado precisamos y qué aspectos son claves en su formación para asumir dicha responsabilidad?}

En cuanto al profesorado es fundamental, en primer lugar, seleccionar a los docentes con las cualidades personales y morales más acordes con su futura responsabilidad. En segundo lugar, dotar a los profesores de una sólida formación humanista, talante cívico, conocimiento de un amplio abanico de metodologías activas y participativas. En definitiva, conseguir docentes formados y preocupados por la formación ética y moral de los estudiantes, que persiguen una escuela más humana, cívica y educadora. Docentes con espíritu de servicio, con sentimiento de "tutor de ciudadanía", respetuosos y valientes frente a los derechos de sus alumnos, capaces de convertirse en palancas de cambio y contrapesos de una sociedad que promulga valores individualistas, injustos y egoístas.

\section{¿Qué aspectos estructurales del sistema educativo debemos replantearnos?}

Por un lado, los contenidos de enseñanza deben transformarse para dar respuesta a los desafíos actuales. El desarrollo de ciertas capacidades personales, morales y sociales deben estar en la base de una nueva educación. Por otro, la metodología docente debe adecuarse a esta realidad, así como las agrupaciones de alumnos y los tiempos de enseñanza. Por tanto, se precisa de cambios en la configuración del sistema y en el curriculum académico.

Entre los aspectos fundamentales a tratar en el currículo de los futuros docentes, se destaca la necesidad de formación tanto en el ámbito específico como en los aspectos pedagógicos imprescindibles para ser maestros. El estudio realizado por Schultz et al (2018) pone en evidencia que se deben manejar tanto las competencias de la propia disciplina como los aspectos relacionados con la práctica pedagógica, estas mismas cuestiones se evidencian en cada uno de los estudios PISA publicados hasta la fecha. 
Respecto del perfil para formar ciudadanos en un mundo global en el siglo XXI, se concreta que el maestro ha de ser un tutor de aprendizaje, y para ser un "tutor de ciudadanía" debe:

1. Adoptar una práctica ética respecto a los valores sociales acorde a la responsabilidad profesional que supone educar para la ciudadanía.

2. Desarrollar en el aula principios democráticos y prácticas cívicas entre el alumnado.

3. Asumir un estilo docente democrático que promueva la participación de los alumnos, a través de la gestión positiva de los espacios y de los tiempos para aprender, atendiendo a la riqueza de la diferencia como garante de una educación universal e inclusiva.

4. Implementar en el currículum académico aprendizajes significativos en torno a la ciudadanía, que respondan a los aspectos cognitivo, afectivo y comportamental.

5. Favorecer en el aula un aprendizaje vivencial, basado en la experiencia e historia personal de cada alumno, que lo sitúe como sujeto responsable de sus acciones en la vida democrática de su entorno.

El siglo XXI necesita ciudadanos libres, responsables, respetuosos, inclusivos, amantes de la verdad y a la altura de la dignidad humana. Por tanto, se precisa de una escuela acorde a este desafío. La educación para la ciudadanía debe hacernos replantear la finalidad actual de la Educación y, para ello, debemos repensar los contenidos curriculares, las metodologías docentes, la formación del profesorado y el modo de seleccionarlo.

\section{Contribuición de los Autores}

Conceptualización de la investigación, Ponce RS, Sarmiento AS, Bertolín AG; Marco teórico, Ponce RS, Sarmiento AS, Bertolín AG; Conclusiones, Ponce RS, Sarmiento AS, Bertolín AG.

\section{Referencias}

ARENDT, H. Between past and future. London: Faber, 1961.

BARBER, M.; MOURSHAEED, M. How the world's best-performing school sistems come out on top. London: Mckinsey \& Company, 2007.

BERNAL, A.; GOZÁlVEZ, V.; BURGUET, M. Construcción ética de la ciudadanía en la actualidad. In: HERNÁNDEZ PRADOS, M. A. (coord.). Educación para la vida ciudadana en una sociedad plural. Murcia: Edit.um, 2017, p. 93-118.

BOKOVA, I. Replantear la educación. ¿Hacia un bien común mundial? París, Unesco, 2015.

BUXARRAIS, R. Educación moral y cívica: Una pedagogía del mirar y del cuidado. In: MÍNGUEZ, R.; ROMERO, E. (coords.). La educación ciudadana en un mundo en transformación: Miradas y propuestas. Barcelona: Octaedro, 2018, p. 141-156.

CHOMSKY, N. La (des)educación. Barcelona: Austral, 2012.

COMISIÓN EUROPEA. Promoting citizenship and the common values of freedom, tolerance and 
non-discrimination through education: Overview of education policy developments in Europe following the Paris Declaration of 17 March 2015. Luxembourg: Publications Office of the European Union, 2016.

COMISIÓN EUROPEA. Comunicación de la Comisión al Parlamento Europeo, al Consejo, al Comité Económico y Social Europeo y al Comité de las Regiones. "Reforzar la identidad europea mediante la Educación y la Cultura”. Gotemburgo: Publicaciones de la Oficina de la Unión Europea, 2017.

CONSEJO DE EUROPA. Carta del Consejo de Europa sobre la educación para la ciudadanía democrática y la educación en derechos humanos. Consejo de Europa, 2010.

DAY, C. Educadores comprometidos. Qué son, qué hacen, por qué lo hacen y lo que verdaderamente importa. Madrid: Narcea, 2018.

DELORS, J. La educación encierra un tesoro. París: Unesco, 1996.

ENKVIST, I. El complejo oficio del profesor. España: FINEO, 2016

FAURE, E. Aprender a ser. La educación del futuro. París: Unesco, 1973.

FUKUDA-PARR, S. Informe sobre Desarrollo Humano 2004. La libertad cultural en el mundo diverso de hoy. Madrid: PNUD, 2004.

FUNDACIÓN CIVES. Estado de la Educación para la Ciudadanía en España. Análisis encuesta. Madrid: Fundación CIVES, 2015.

GARCÍA ARETIO, L.; RUÍZ CORBELLA, M.; GARCÍA BLANCO, M. Claves para la educación. Actores, agentes y escenarios en la sociedad actual. Madrid: Narcea, 2011.

GARCÍA RAGA, L.; LÓPEZ MARTÍN, R. La convivencia escolar y la construcción de la ciudadanía. Balance retrospectivo y desafíos de futuro. Bordón, v. 66, n. 2, p. 93-106, 2014.

GIROUX, H. Hacia una pedagogía de la esperanza educada bajo el capitalismo de casino. Pedagogía y Saberes, v. 50, p. 153-158, 2019. https://doi.org/10.17227/pys.num50-9508

GONZÁLEZ BERTOLÍN, A.; SANZ, R. De la relevancia de las reformas educativas en la evolución de la formación del profesorado de Educación Secundaria. Revista Currículum y Formación del Profesorado, v. 18, n. 1, p. 367-381, 2014.

GONZÁLEZ BERTOLÍN, A.; SANZ, R. Desafíos y tensiones del sistema educativo del siglo XXI. Una mirada desde la pedagogía. Crónica - Revista Científico Profesional de la Pedagogía y la Psicopedagogía, v. 1, p. 7-19, 2016.

GUICHOT, V. Tolerancia, una virtud cívica clave en una educación para la ciudadanía activa, compleja e intercultural. Bordón, v. 64, n. 4, p. 35-47, 2012.

HARGREAVES, A. Enseñar en la sociedad del conocimiento. Barcelona: Octaedro, 2012.

LLANO, A. Humanismo cívico y formación ciudadana. In: NAVAL, C.; LASPALAS, J. (eds.). La educación cívica hoy. Una aproximación interdisciplinar. Navarra: Eunsa. 2007, p. 97-100.

LÓPEZ-LUJÁN, E. Dimensiones para evaluar una buena práctica educativa: El Cuestionario sobre Competencias del Profesorado (CCPESII). Edetania, v. 54, p. 53-71, 2018. 
MARINA, J. A. El bosque pedagógico. Lo que los padres y docentes deben saber. Barcelona: Ariel, 2017.

MARINA, J. A.; PELLICER, C.; MANSO, J. Libro Blanco de la profesión docente y su entorno escolar, 2015. Disponible en: http://www.funciva.org/wp-content/uploads/2016/11/Libro-blanco-de-la-profesi\%C3\%B3ndocente.pdf, Acceso en: 3 oct. 2015.

MARTIN, M. O.; MULLIS, I. V. TIMSS and PIRLS 2011: Relationships among reading, mathematics, and science achievement at the fourth grade - Implications for early learning. United States: International Association for the Evaluation of Educational Achievement, 2013.

MECD [Ministerio de Educación, Cultura y Desporte]. TALIS 2013. Estudio Internacional sobre la Enseñanza y el Aprendizaje. Informe Internacional. Boletín de Educación, p.1-4, 2014.

MEFP [Ministerio de Educación y Formación Profesional]. TALIS 2018. Estudio Internacional de la Enseñanza y del Aprendizaje. Informe español. Madrid: Ministerio de Educación y Formación Profesional, 2019.

MEIRIEU, P.; MONTENEGRO, P. A. Riquezas y límites del enfoque por “competencias” del ejercicio de la profesión docente hoy. Pedagogía y Saberes, v. 50, p. 97-108, 2019.

MIGUEL, V.; BLANCO, J. L. Neurociencia en las aulas. Su aplicación en los procesos de aprendizaje. Madrid: Wolters Kluwer, 2019.

MÍNGUEZ, R.; ROMERO, E. Por la transformación de la educación ciudadana. In: MÍNGUEZ, R.; ROMERO, E. (coords.). La educación ciudadana en un mundo en transformación: Miradas y propuestas. Barcelona: Octaedro, 2018, p. 7-16.

MORÍN, E. Los siete saberes necesarios para la educación del futuro. México: Unesco, 2015.

NAVAL, C.; FUENTES, J. L.; QUINTANILLA, I. La educación para la ciudadanía global. Buenas teorías que orientan buenas prácticas. In: HERNÁNDEZ PRADOS, M. A. (coord.). Educación para la vida ciudadana en una sociedad plural. Murcia: Edit.um, 2017, p. 243-271.

NOVELLA, A. M. et al. El concepto de ciudadanía construido por jóvenes que vivieron experiencias de participación infantil. Bordón, v. 65, n. 3, p. 39-108, 2013.

NUSSBAUM, M. La monarquía del miedo. Una mirada filosófica a la crisis política actual. Barcelona: Paidós, 2019.

OCDE [Organization for Economic Co-operation and Development]. Do teacher-student relations affect students' well-being at school. PISA in Focus, v. 50, p.1-4, 2015.

OECD [Organization for Economic Co-operation and Development]. TALIS 2018. Results (Volume I). Teachers and School Leaders as Lifelong Learners. París: OECD Publishing, 2019.

PÉREZ, C. Educación en valores para la ciudadanía. Estrategias y técnicas de aprendizaje. Bilbao: Desclée, 2016.

PRING, R. Una filosofía de la educación políticamente incómoda. Madrid: Narcea, 2016.

PUIG, M.; MORALES, J. A. La formación de ciudadanos: Conceptualización y desarrollo de la competencia social y cívica. Educación XX1, v. 18, n. 1, p. 259-282, 2015. https://doi.org/10.5944/educxx1.18.1.12332

ROBINSON, K. Escuelas creativas. La revolución que está transformando la educación. Barcelona: Grijalbo, 2015. 
SANZ, R.; SERRANO, A. El desarrollo de capacidades en la educación. Una cuestión de justicia social. Sinéctica - Revista Electrónica de Educación, v. 46, p. 23-38, 2016.

SANZ, R.; SERRANO, A. ¿La educación cambia? Repensando el sentido y finalidad de una escuela para todos. Teoría de la Educación - Revista Interuniversitaria, v. 29, n; 2, p. 167-184, 2017.

SCHULZ, W. et al. ICCS 2009. Technical report. Amsterdam: International Association for the Evaluation of Educational Achievement, 2011.

SCHULZ, W. et al. International Civic and Citizenship Education Study 2016. Assessment Framework. Amsterdam: International Association for the Evaluation of Educational Achievement, 2016.

SCHULZ, W. et al. Becoming citizens in a changing world: IEA International Civic and Citizenship Education Study 2016 International Report. Amsterdam: Springer, 2018.

SCHWARTZ, S.; POLLISHUKE, M. Aprendizaje activo. Una organización de la clase centrada en el alumnado. Madrid: Narcea, 2015.

UNESCO. The World Needs Almost 69 Million New Teachers to Reach the 2030 Education Goals. In Focus, n. 39, 2016. Disponible en: http://uis.unesco.org/sites/default/files/documents/fs39-the-world-needs-almost69-million-new-teachers-to-reach-the-2030-education-goals-2016-en.pdf, Acceso en: 26 abr. 2017.

UNESCO. Measuring Global Citizenship Education. A Collection of Practices and Tools. Center For Universal Education at Brookings, 2017. Disponible en: https://www.brookings.edu/wp-content/uploads/2017/04/ global_20170411_measuring-global-citizenship.pdf, Acceso en: 15 feb. 2018.

\section{Sobre los autores}

Roberto Sanz Ponce es doctor en Ciencias de la Educación por la Universidad de Valencia. Profesor de la Universidad Católica de Valencia. Líneas de investigación: Formación del Profesorado y Competencias Docentes

Ángela Serrano Sarmiento es doctora en Ciencias de la Educación por la Universidad de Valencia. Profesora de la Universidad Católica de Valencia. Líneas de investigación: Formación del Profesorado, Violencia Escolar y Resolución de Conflictos

Aurelio González Bertolín es doctor en Ciencias de la Educación por la Universidad de Valencia. Profesor de la Universidad Católica de Valencia. Líneas de investigación: Formación del Profesorado, Política y Legislación Educativa. 